\title{
Nuclear Magnetic Resonance Shared Resource
}

National Cancer Institute

\section{Source}

National Cancer Institute. Nuclear Magnetic Resonance Shared Resource. NCI

Thesaurus. Code C39478.

Nuclear Magnetic Resonance (NMR) Shared Resource serves cancer research projects in the field of structural biology as well as in synthesis and analysis of complex natural products and synthetic molecules, including conformational analysis and relationship of structure and biological activity. 